\title{
Recommended survey designs for occupancy modelling using motion-activated cameras: insights from empirical wildlife data
}

Motion-activated cameras are a versatile tool that wildlife biologists can use for sampling wild animal populations to estimate species occurrence. Occupancy modelling provides a flexible framework for the analysis of these data; explicitly recognizing that given a species occupies an area the probability of detecting it is often less than one. Despite the number of studies using camera data in an occupancy framework, there is only limited guidance from the scientific literature about survey design trade-offs when using motion-activated cameras. A fuller understanding of these trade-offs will allow researchers to maximise available resources and determine whether the objectives of a monitoring program or research study are achievable. We use an empirical dataset collected from 40 cameras deployed across 160 $\mathrm{km}^{2}$ of the Western Slope of Colorado, USA to explore how survey effort (number of cameras deployed and the length of sampling period) affects the accuracy and precision (i.e. error) of the occupancy estimate for ten mammal and three virtual species. We do this using a simulation approach where species occupancy and detection parameters were informed by empirical data from motion-activated cameras. A total of 54 survey designs were considered by varying combinations of sites (10-120 cameras) and occasions (20-120 survey days). Our findings demonstrate that increasing total sampling effort generally decreases error associated with the occupancy estimate, but changing the number of sites or sampling duration can have very different results, depending on whether a species is spatially common or rare (occupancy $=\psi$ ) and easy or hard to detect when available (detection probability = $p$ ). For rare species with a low probability of detection (i.e., raccoon and spotted skunk) the required survey effort includes maximizing the number of sites and the number of survey 
days, often to a level that may be logistically unrealistic for many studies. For common species with low detection (i.e., bobcat and coyote) the most efficient sampling approach was to increase the number of occasions (survey days). However, for common species that are moderately detectable (i.e., cottontail rabbit and mule deer), occupancy could reliably be estimated with comparatively low numbers of cameras over a short sampling period. We provide general guidelines for reliably estimating occupancy across a range of terrestrial species (rare to common: $\psi=0.175-0.970$, and low to moderate detectability: $p=0.003-$ 0.200) using motion-activated cameras. Wildlife researchers/managers with limited knowledge of the relative abundance and likelihood of detection of a particular species can apply these guidelines regardless of location. We emphasize the importance of prior biological knowledge, defined objectives and detailed planning (e.g. simulating different study-design scenarios) for designing effective monitoring programs and research studies. 


\section{Recommended survey designs for occupancy modelling using motion-activated \\ 2 cameras: insights from empirical wildlife data}

3 Graeme Shannon ${ }^{1}$, Jesse Lewis ${ }^{1,2} \&$ Brian D. Gerber ${ }^{3}$

$4 \quad{ }^{1}$ Department of Fish, Wildlife, and Conservation Biology, Colorado State University, Fort

5 Collins, Colorado, United States of America

$6{ }^{2}$ Graduate Degree Program in Ecology, Colorado State University, Fort Collins, Colorado, United

7 States of America

$8{ }^{3}$ Colorado Cooperative Fish and Wildlife Research Unit, Department of Fish, Wildlife and

9 Conservation Biology, Colorado State University, Fort Collins, CO 80523, United States of

10 America

11 Running Title: Camera survey design

12 Address for Correspondence:

13 Dr Graeme Shannon

14 Department of Fish, Wildlife, and Conservation Biology

15 Colorado State University

16 Fort Collins, CO 80523-1474

17 USA

18 Email: graeme.shannon@ colostate.edu 


\section{Abstract}

20 Motion-activated cameras are a versatile tool that wildlife biologists can use for sampling wild

21 animal populations to estimate species occurrence. Occupancy modelling provides a flexible

22 framework for the analysis of these data; explicitly recognizing that given a species occupies an

23 area the probability of detecting it is often less than one. Despite the number of studies using

24 camera data in an occupancy framework, there is only limited guidance from the scientific

25 literature about survey design trade-offs when using motion-activated cameras. Our aim was to

26 provide a fuller understanding of these trade-offs to enable researchers with varying backgrounds

27 in ecological modelling and statistics to maximize available resources and determine whether the

28 objectives of a monitoring program or research study are achievable. We use an empirical dataset

29 collected from 40 cameras deployed across $160 \mathrm{~km}^{2}$ of the Western Slope of Colorado, USA.

30 Specifically, we use these empirical data to estimate species occupancy and detection parameters

31 from motion-activated cameras that are then used in a simulation exercise. This approach

32 provides a clear link between field data and the simulations that explore how survey effort

33 (number of cameras deployed and the length of sampling period) affects the accuracy and

34 precision (i.e. error) of the occupancy estimate for ten mammal and three virtual species. A total

35 of 54 survey designs were considered by varying combinations of sites (10-120 cameras) and

36 occasions (20-120 survey days). Our findings demonstrate that increasing total sampling effort

37 generally decreases error associated with the occupancy estimate, but changing the number of

38 sites or sampling duration can have very different results, depending on whether a species is

39 spatially common or rare (occupancy $=\psi$ ) and easy or hard to detect when available (detection

40 probability $=p$ ). For rare species with a low probability of detection (i.e., raccoon and spotted

41 skunk) the required survey effort includes increasing the number of sites and the number of

42 survey days, often to a level that may be logistically unrealistic for many studies. For common

43 species with low detection (i.e., bobcat and coyote) the most efficient sampling approach was to

44 increase the number of occasions (survey days). However, for common species that are

45 moderately detectable (i.e., cottontail rabbit and mule deer), occupancy could reliably be

46 estimated with comparatively low numbers of cameras over a short sampling period. We provide 
47 general guidelines for reliably estimating occupancy across a range of terrestrial species (rare to

48 common: $\psi=0.175-0.970$, and low to moderate detectability: $p=0.003-0.200$ ) using motion-

49 activated cameras. Results from our study have wide applicability to camera studies conducted

50 across a range of environments and taxa, which can be used by wildlife researchers/managers

51 with limited knowledge of the relative abundance and likelihood of detection of a particular

52 species. We emphasize the importance of prior biological knowledge, defined objectives and

53 detailed planning (e.g. simulating different study-design scenarios) for designing effective

54 monitoring programs and research studies.

\section{Introduction}

56 Estimating the distribution of a species or suite of species across the landscape provides wildlife

57 biologists with crucial information for monitoring and conserving animal populations (Noon et

58 al. 2012). It is also a key criteria for global conservation initiatives such as the International

59 Union for Conservation of Nature red list (http://www.iucnredlist.org/), which has been used to

60 track the change in extinction risk of threatened species over time (Di Marco et al. 2014).

61 Motion-activated cameras are one of the fastest growing techniques for surveying a wide range of

62 terrestrial animals, particularly those that are rare, elusive or cryptic (O'Connell et al. 2011; Jamie

63 2012). The advancement of affordable and reliable digital camera technology in combination

64 with infrared triggers and time delays has enabled biologists to deploy multiple cameras

65 simultaneously to collect data in an efficient and minimally invasive manner. These data have

66 allowed biologists to investigate a diversity of ecological and conservation driven questions,

67 relating to species abundance (Gerber et al. 2010) and density (O’Brien \& Kinnaird 2011),

68 animal behaviour (Maffei et al. 2011), survival (Gardner et al. 2010), temporal activity (Ridout \&

69 Linkie 2009), and landscape-level occurrence (Thorn et al. 2009). Cameras are typically more

70 efficient than traditional sampling methods (e.g. direct observation, radio telemetry) as

71 continuous data can simultaneously be collected on multiple species (e.g. large bodied

72 carnivores; O'Brien \& Kinnaird 2011). The field deployment can be standardised and readily

73 replicated, enabling researchers to monitor whether there are changes in the occurrence of target

74 indicator species over both time and space (Ahumada et al. 2011; Ahumada, Hurtado \& Lizcano

75 2013). 
77 occurrence, offers a very useful analytical framework for analysing data collected from motion-

78 activated cameras (O'Connell \& Bailey 2011). Occupancy models explicitly recognize that given

79 a species occurs in an area, the probability of detecting it on a single survey is often less than one.

80 This potential source of bias is addressed by using repeat sampling across multiple sites, enabling

81 detection probability to be calculated and incorporated in the occupancy estimate (MacKenzie et

82 al. 2002, 2006). Among the key benefits of occupancy studies is that detection/non detection data

83 can generally be collected with greater ease and cost effectiveness for a greater number of species

84 than the more detailed demographic data that are commonly required for estimates of abundance

85 and density (Jones 2011). As a result, occupancy modelling is increasingly used to evaluate

86 species distribution (Long et al. 2010), habitat use (Betts et al. 2008) and population dynamics

87 (MacKenzie et al. 2010). The results from these studies and monitoring programs have the

88 potential to be used by wildlife managers and conservation practitioners to determine changes in

89 the distribution of key animal populations as well as strengthening future demographic

90 predictions (Jones 2011; Noon et al. 2012).

91 There are clear advantages to using motion-activated cameras in occupancy studies;

92 nevertheless, in common with other survey techniques, the efficacy of these studies and

93 monitoring programs relies on appropriate and detailed survey design. These considerations

94 include deciding upon what time period to sample, the sampling length, and the number of

95 cameras to deploy, which is dependent on the target species and the type of inference that is

96 sought (MacKenzie et al. 2006). For community studies, it is important to recognize that an

97 optimal survey for one species may not be so for another; designing a community-level

98 occupancy study will likely incur trade-offs in efficiency and the scope of inference depending on

99 how well the sampling period and duration coincides with a meaningful biological time frame for

100 each species. Research studies and monitoring programs that are initiated without well-defined

101 objectives and rigorous survey design increase the likelihood of returning results that are

102 insufficient to make meaningful inference on the species or system of interest (Yoccoz, Nichols

103 \& Boulinier 2001; Kéry \& Schmid 2004; Mattfeldt, Bailey \& Grant 2009). Moreover, as

104 conservation and research programs are often limited by the availability of funding, it is crucial

105 that surveys are justified in terms of the costs and benefits of acquiring the data (Nichols \&

106 Williams 2006; McDonald-Madden et al. 2010). 
107 Estimates of population parameters often require spatial and temporal replication; in

108 occupancy studies this generates a trade-off in survey effort between the number of sites to

109 sample and the number of replicates to conduct at each site (MacKenzie et al. 2002; 2003; Tyre

110 et al. 2003; MacKenzie et al. 2006). A further consideration is that occupancy is assumed to be

111 static during the designated sampling period (assumption of closure; MacKenzie et al. 2002), and

112 the length of this period may vary depending upon the species and biological timeframe of

113 interest (e.g. the breeding season; Webber, Heath \& Fischer 2013). There are a number of studies

114 that provide theoretical background to study design using an occupancy-modelling framework,

115 highlighting the importance of balancing temporal and spatial replication to most efficiently

116 achieve defined objectives (MacKenzie \& Royle 2005; Bailey et al. 2007; Guillera-Arroita,

117 Ridout \& Morgan 2010; Guillera-Arroita \& Lahoz-Monfort 2012). However, there are few

118 studies that have used empirical data from a suite of species to evaluate the effects of varying the

119 number of sites and occasions on the accuracy and precision of occupancy estimates. Moreover,

120 the majority of research exploring the effective use of motion-activated cameras has focussed on

121 1) comparing cameras with other sampling approaches (Rovero \& Marshall 2009; Janečka et al.

122 2011), 2) investigating sampling efficiency as a function of biological parameters (e.g. species,

123 sex, habitat, and season; Larrucea et al. 2007; Kelly \& Holub 2008), and 3) evaluating alternative

124 approaches to species inventories (Tobler et al. 2008; Si, Kays \& Ding 2014). However, a recent

125 analysis conducted on a dataset of avian and mammalian scavengers in sub-arctic environments

126 provided the first detailed discussion of guidelines to determine the optimal survey design for

127 estimating occupancy using empirical data collected from time-triggered cameras (Hamel et al.

128 2013).

129 The aim of this study is to provide practitioners who may not have expertise in study

130 design, ecological modeling, or statistics with detailed examples of how survey design influences

131 the accuracy and precision (i.e. error) of occupancy estimates across a range of mammal species

132 using an extensive motion-activated camera dataset. Empirical data allows us to make the

133 tangible connection between values of detection probability, occupancy and the outcomes of the

134 survey design analysis for a given species. In addition, occupancy and detection values for

135 species targeted in motion-activated camera studies (e.g. small-large bodied mammals), as well as

136 sampling length are often in combinations that are not typically evaluated in other study design

137 papers. In fact it is common that per occasion, detection probability is often much lower than 
138 values that are usually evaluated $(<0.2$ or even $<0.1)$. Furthermore, we envisage that the survey

139 designs that we test in this paper can apply to research that uses motion-activated cameras to

140 study tax across a diverse range of terrestrial ecosystems.

141 Our specific research objective is to evaluate how varying the number of sampling sites

142 (10-120 cameras) in combination with the number of occasions (20-120 survey days) influences

143 the error associated with estimating occupancy for 10 mammal species and three 'virtual' species.

144 These thirteen species characterize a range of comparatively rare-to-common species with low-

145 to-moderate detection probability, which are typically encountered during camera sampling of

146 terrestrial mammals. Using these results, we provide recommendations and general guidelines

147 that can be used by wildlife practitioners to design and implement studies to evaluate mammal

148 occurrence using motion-activated cameras.

\section{Methods}

\section{Study Site}

151 The study site was located on the Western Slope (WS) of Colorado, USA on the Uncompahgre

152 Plateau near the towns of Montrose and Ridgway (Figure 1). The area was characterized by

153 mesas, canyons, and ravines, with elevations ranging from $1800 \mathrm{~m}$ to $2600 \mathrm{~m}$ and annual

154 precipitation of $430 \mathrm{~mm}$ arriving primarily from winter snows and summer thunderstorms

155 (NOAA National Climatic Data). The vegetation communities were dominated by pinyon pine

156 (Pinus edulis) and juniper (Juniperus osteosperma), ponderosa pine (Pinus ponderosa), aspen

157 (Populus tremuloides), gambel oak (Quercus gambelii), and big sagebrush (Artemesia

158 tridentata). The WS had extensive areas of undeveloped natural habitat managed by the Bureau

159 of Land Management, US Forest Service, and private landowners. Paved and unimproved roads

160 occurred throughout the WS. The WS has a history of ranching with some private ranches

161 converted into exurban and rural housing developments.

\section{Study Design}

163 We deployed 40 motion-activated cameras across two survey grids totaling $160 \mathrm{~km}^{2}$, with

164 individual camera sites spaced approximately $2 \mathrm{~km}$ apart. The sampling design was specifically

165 focused on surveying mountain lions (Puma concolor) and bobcats (Lynx rufus) with cameras

166 placed along game trails, hiking trails, and secondary dirt roads. The placement of cameras along 
167 likely travel routes of mammals is common in camera studies and often leads to detecting a

168 diverse assemblage of the mammalian community (O'Connell, Nichols \& Karanth 2011). We

169 checked cameras approximately every two weeks to replace memory cards and batteries if

170 required. The sampling approach was passive in that we did not use attractants (i.e., sight, sound,

171 scent) to lure animals to the camera location. Motion-activated cameras operated from August 21

172 to December 13, 2009. As the study involved non-invasive sampling using motion-activated

173 cameras there was no requirement for institutional review of the proposed research. Data

174 collection was funded by a grant from the National Science Foundation (NSF EF-0723676).

\section{Data and statistical analyses}

176 We took a two-step approach in our analyses. First, the empirical data collected from motion-

177 activated cameras were used to estimate daily detection probabilities and occupancy estimates for

178 a range of terrestrial mammal species with closure assumed for the entire sampling period (i.e. no

179 changes in occupancy). Second, this information was used in simulations to evaluate optimal

180 survey design approaches for the different species. Photographic data were analysed for ten

181 mammal species (see Figure 2; the number of photographs are provided in parentheses), raccoons

182 (Procyon lotor: 8), spotted skunks (Spilogale putorius: 25), mountain lions (83), black bears

183 (Ursus americanus: 96), gray foxes (Urocyon cinereoargenteus: 144), coyotes (Canis latrans:

184 192), elk (Cervus canadensis: 196), bobcats (225), cottontail rabbits (Sylvilagus nuttallii: 1267)

185 and mule deer (Odocoileus hemionus: 1753).

186 For the purpose of our study, a sampling occasion was defined as a $24 \mathrm{~h}$ period, which we

187 refer to as a survey day. However, values of detection probability are dependent upon the length

188 of the sampling occasion and researchers will often employ sampling occasions that are measured

189 in weeks rather than days (Ellis, Ivan \& Schwartz 2013). Thus, if the daily detection probability

190 is 0.03 , we can recalculate $p$ using the $p^{*}$ formula $\left(p^{*}=1-(1-p)^{\mathrm{s}}\right)$ such that at 1 week $p=0.19$,

1912 weeks $=0.35$ and so on. It is also important to note that optimal design for continuous sampling

192 protocols has also recently been explored, which does not rely on discretizing the data (Guillera-

193 Arroita et al. 2011).

194 Species-specific detection histories were generated for each of the 40 cameras across the

195 four-month sampling period (except black bears, where only the first two months of data were

196 used due to animals hibernating in November and December). For a given species, detection 
197 histories provide a record of whether the species was detected (1) or not detected (0) on each

198 survey day for each camera location (40 detection histories for each species). These detection

199 histories were then used to estimate a constant occupancy $\left(\psi_{i}\right)$ and constant detection probability

$200\left(p_{i}\right)$ for each species $i$ from $i=1,2, \ldots, 10$ using the single-species, single-season occupancy

201 model (MacKenzie et al. 2002). In addition, we created three 'virtual' species that were not

202 characterized by our empirical data, but that researchers might encounter, to provide examples

203 where daily detection probability is relatively high $(>0.1)$, while occupancy levels are low to

204 moderate ( $\leq 0.6$; Figure 3 ). We constructed models using the RMark package (Laake and Rexstad

205 2013) in the R programming language (R Development Core Team 2013), which interfaces with

206 Program MARK (White and Burnham 1999). The resulting 13 species provide a range of daily

207 detection probabilities and occupancy estimates that are typical for mammals surveyed with

208 motion-activated cameras. The species are classified into seven distinct groups ranging from rare

209 and hard to detect species (i.e., raccoon and spotted skunk) to common detectable species (i.e.,

210 cottontail rabbit and mule deer; see Figure 3).

\section{Simulation approach}

212 The occupancy and detection probabilities estimated from the empirical data were used to explore

21354 different scenarios for each individual species, using a combination of the number of survey

214 days (occasions: $S=(20,40,60,80,100,120))$ and number of cameras (sites: $N=(10,20,30$,

$21540,50,60,70,80,120)$ ). For each species $i$, a detection history was created that is $N$ x $S$, where

216 each site $j$ from $j=1,2, \ldots \mathrm{N}$ is considered to be occupied or not following a Bernoulli process

217 with probability $\psi_{i}$; we then determined whether a species was detected or not at occupied sites

218 for each occasion $t$, from $t=1,2, \ldots, S$, following a Bernoulli process with probability $p_{i}$. In total,

2191000 sets of detection histories were simulated for each species and each combination of $S$ and $N$

220 and error was calculated using root mean squared error (RMSE) as:

221

$$
\begin{gathered}
E[\dot{\iota} 2]=\sqrt{\operatorname{Var}(\hat{\psi}-\psi)+(\operatorname{Bias}(\hat{\psi}, \psi))^{2}} \\
R M S E=\sqrt{i}
\end{gathered}
$$

222 Given our simulation setting, the generating and estimating model are equivalent, such 223 that bias will generally be low, except for cases of small-sample bias when survey effort is very 
224 low. Thus, the majority of error in RMSE across most scenarios is due to the variance, to the

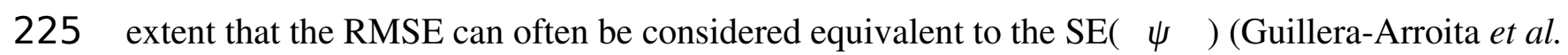

226 2010; Gardner et al. 2010). For each scenario, the RMSE was plotted for the number of sites

227 (cameras) and the length of the sampling period (days). This was repeated for each of the 13

228 species. To assess the optimal survey design approach, three different RMSE target values were

229 selected representing differing levels of error; these included RMSE of $0.15,0.10$ and 0.075 . It is

230 important to note that an RMSE of 0.15 will result in estimates that will likely be imprecise. This

231 level of error is included for the purposes of comparison, rather than a suggested target value that

232 we recommend should be used in study design. For the purpose of our analysis, we weight

233 occasions (days) and sites (cameras) equally to obtain an optimal solution (but see Table S1).

234 RMSE values of less than 0.1, reflect thresholds used in the wildlife occupancy literature and are

235 considered realistic for determining shifts in occupancy over time and space (MacKenzie \&

236 Royle 2005; Guillera-Arroita et al. 2010). The optimal survey design (combination of cameras

237 and number of survey days) was then selected as the minimum survey effort required for each

238 species that enabled the estimate of occupancy to be calculated within the desired level of error.

239 Ideally, the lowest level of RMSE is preferred, but this may be logistically unachievable for some

240 species.

\section{Results}

242 Increasing survey effort reduced RMSE for all species (Figure 4). However, the optimal

243 combination of the number of sites (motion-activated cameras) and occasions (survey days)

244 varied widely across the 13 species. It was also commonly found that the reduction in RMSE as a

245 result of either increasing the number of cameras or sampling duration would eventually stabilise

246 and would offer limited benefit to further increases in survey effort. Overall, the minimum

247 amount of sampling effort (combination of motion-activated cameras and survey days) required

248 to obtain an acceptable level of error was reduced with increasing daily probability of detection.

249 Of the species of interest, raccoons had the lowest levels of occupancy coupled with

250 extremely low levels of detection - representing a very rare and hard to detect species in our

251 study area (Figure 3). Even the maximum survey effort (120 motion-activated cameras operating

252 over 120 days) totaling 14400 survey days could not guarantee a reliable occupancy estimate for

253 raccoons; approximately $40 \%$ of the simulations failed to numerically converge at a maximum- 
254 likelihood estimate due to a lack of data. Spotted skunks were also rare and difficult to detect

255 (Figure 3), however intensive sampling was able to reliably estimate occupancy, requiring 2000

256 survey days (e.g. a sampling period of 100 days with 20 cameras) for the highest level of

257 acceptable error $(\mathrm{RMSE}=0.15)$ and 5000 survey days for the lowest threshold of error $(\mathrm{RMSE}=$

258 0.075). These results demonstrate the substantial effort that is required to accurately document

259 the presence of rare and elusive species (Figure 4 and Table 1).

260 Species that were fairly common with intermediate levels of occupancy but with low

261 detection probabilities (i.e., elk and mountain lion; Figure 3) also required intensive sampling that

262 used a comparatively large number of both sites and occasions. When the number of occasions

263 increased from 20 to approximately 80 survey days there was a substantial decrease in RMSE for

264 mountain lion and elk, while estimation error was only further improved by including additional

265 sites to the study design (Table $1 \&$ Figure 4).

266 For those species with high occupancy $(>0.8)$ and relatively low levels of detection (i.e.,

267 coyote and bobcat; Figure 3), the overall survey effort required to achieve a desired level of error

268 is significantly reduced (compared to spotted skunk, mountain lion and elk). Indeed, increasing

269 the number of occasions at comparatively few sites returns a reliable estimate (Table $1 \&$ Figure

270 4). For example, 10 motion-activated cameras proved sufficient to achieve the desired RMSE of

$2710.15,0.10$ and 0.075 for bobcats, with the reduction in error achieved by including a greater

272 number of survey days (Table 1). As detection probability increases for more common species

273 (i.e., black bear and gray fox), sampling periods over 40 survey days provide no substantial

274 reduction in associated error and argue against continuing the survey. We found the optimal

275 approach for these common species is to sample between 30-50 sites (cameras) over a period of

27640 survey days depending upon the level of error that is acceptable (see Table 1).

277 For species with comparatively high levels of daily detection $(\geq 0.12$; mule deer, cottontail

278 rabbit and virtual species 1-3), there is only a limited reduction in error associated with

279 lengthening the survey beyond approximately 30 days, particularly for species with moderate to

280 high estimates of occupancy (i.e., virtual species 2, mule deer and cottontail rabbit; Figure 3).

281 Precise occupancy estimates for these species can be achieved with relatively few cameras (see

282 Table 1). Nonetheless, improving upon these estimates generally requires adding additional sites

283 (cameras) rather than more survey days (Table $1 \&$ Figure 4). For example, a RMSE of 0.085 can

284 be achieved for cottontail rabbits using only 10 cameras and 20 days of sampling. Further 
285 reductions in error cannot be achieved by lengthening the sampling period, with 120 survey days 286 returning an RSME of 0.083 (Figure 4); however deploying additional cameras can substantially

287 reduce error (Figure 4). Virtual species 1 and 3 both have low occupancy estimates but

288 comparatively high probabilities of detection (Figure 3). An intermediate level of survey effort is

289 required in order to achieve the most efficient sampling approach, which depending upon the

290 desired level of precision, balances the number of cameras (20-50) with survey days (20-60;

291 Table 1).

292 Reducing RMSE by increasing sampling length depends on the probability of detecting

293 the species at an occupied site at least once over the entire sampling duration. We calculated this

294 probability as $p^{*}\left(p^{*}=1-(1-p)^{\mathrm{s}}\right)$, where $p$ is the daily detection probability and $\mathrm{S}$ is the number

295 sampling occasions. When $p^{*}$ is greater than 0.9 there was very little reduction in RMSE by

296 further increasing the number of sampling occasions (see Table 2).

297 Our simulation results reveal broad patterns in survey design when using motion-activated

298 cameras that depend upon how easy a species is to detect and how common it is across the

299 landscape (Figure 5). Rare species with low detection require an intensive sampling approach that

300 combines multiple camera sites and occasions to reliably calculate an occupancy estimate,

301 whereas the best strategy for more common species with low levels of detection involves

302 increasing the number of survey days (occasions) at comparatively few sites ( $\leq 30$ cameras). As

303 detection probability increases, the overall survey effort required to achieve an acceptable level

304 of precision in occupancy is reduced. Species that exhibit moderate detectability but remain

305 comparatively rare generally require an intermediate number of cameras and greater survey

306 lengths to improve precision, compared with common and detectable species that can be

307 surveyed precisely with relatively few sites and short sampling periods (Figure 5).

\section{Discussion}

309 Reliable indicators that document landscape-to-regional biodiversity are urgently needed given

310 the global extinction crisis ( Mace \& Baillie 2007; Butchart et al. 2010). Motion-activated

311 cameras can provide scientists and wildlife managers with a very powerful tool for recording the

312 presence and occupancy of a diverse range of species (O'Connell \& Bailey 2011; Ahumada,

313 Hurtado \& Lizcano 2013), particularly given the advances in storage, reliability and battery life

314 of the latest devices (Jamie 2012). Nevertheless, in common with other ecological monitoring and 
315 research programs, successful sampling strategies rely on detailed study design. Indeed, pursuing

316 an optimal survey design allows available time and resources to be most efficiently used, while

317 also providing guidance as to whether the objectives are achievable and justified given potential

318 funding constraints (McDonald-Madden et al. 2010).

319 Survey design using an occupancy framework has been explored in a number of primary

320 research papers, which given particular detection and occupancy values have provided detailed

321 theoretical insights into the trade-off between the number of sites and the number of occasions

322 (MacKenzie \& Royle 2005; Bailey et al. 2007; Guillera-Arroita, Ridout \& Morgan 2010;

323 Guillera-Arroita \& Lahoz-Monfort 2012). Furthermore, a number of excellent simulation tools

324 are freely available for exploring survey design (e.g. GENPRES: Bailey et al. 2007, SODA:

325 Guillera-Arroita, Ridout \& Morgan 2010). It is important to note that the purpose of our study

326 was not to advance these analytical methods, or indeed provide a detailed statistical explanation

327 of the simulation results. Instead, we have used an occupancy framework to specifically analyse a

328 motion-activated camera dataset to provide a range of realistic scenarios that outline how survey

329 design and effort varies depending upon the species of interest. Although a comparatively

330 straightforward approach from a quantitative perspective, we believe that the value of our study

331 lies in providing guidance and key worked examples to a broad audience of wildlife practitioners

332 that may be planning to use cameras for monitoring or research initiatives. Furthermore, cameras

333 present a useful tool for surveying rare species across the landscape, and as such, the inclusion of

334 species with very low detection and occupancy demonstrate the level of effort required to achieve

335 desired objectives for these taxa. Our study follows on from recent research that has explored

336 camera survey design in terms of species richness (Si et al. 2014) and time-triggered devices

337 (Hamel et al. 2013).

338 We found substantial differences in optimal survey designs across mammal species from

339 our study area, concurring with MacKenzie \& Royle (2005) who highlighted that surveying as

340 many sites as possible is not the most efficient approach to reducing overall occupancy

341 estimation error. Instead, obtaining a reliable and efficient occupancy estimate requires tailoring

342 the study design to the species of interest. Unsurprisingly, the most challenging taxa to develop

343 an appropriate survey design for were the rare and hard to detect species (e.g. raccoons). Even

344 with considerable survey effort it was challenging, if not impossible, to reliably estimate

345 occupancy based on our criteria of RMSE. If the goal of a study is to estimate the occupancy of a 
346 rare species that is difficult to detect, it may be necessary to reposition the cameras to target

347 specific taxa (Karanth \& Nichols 2002), use baits or lures (Thorn et al. 2009) or employ multiple

348 methods (e.g., cameras, sign surveys: Magoun et al. 2011). Even if each method individually has

349 a low probability of detection, the combined effect of all methods incorporated together will be

350 greater, and thus potentially lead to a reliable occupancy estimate. Such an approach can be

351 carried out using multi-scale occupancy models, which allow data to be incorporated from

352 multiple detection methods while permitting estimation of occupancy across different spatial

353 scales (Nichols et al. 2008).

354 Alternatively, for threatened and endangered species it may be more appropriate to forego

355 estimating species occurrence and simply try to determine if the species is present in the area of

356 interest (MacKenzie et al. 2006; Si et al. 2014). We provide an example in the supplemental

357 information evaluating the probability and financial cost of photographing a very rare species $(\psi$

$358=0.05$ and $p=0.05$ ) at least once (see Table S2 \& Spreadsheet S1). Meanwhile, for common and

359 highly detectable species (e.g., cottontail rabbits and mule deer), relatively few motion-activated

360 cameras and survey days are necessary to provide accurate and precise occupancy estimates. As

361 such, rapid assessment surveys could be routinely used to monitor these species relatively

362 inexpensively, while taxa that are comparatively rare across the landscape but yet remain highly

363 detectable (e.g., virtual species 1) require greater survey effort, and are therefore best sampled

364 using an intermediate number of sites and survey days.

365 Our results demonstrated that conducting extended survey periods to estimate occupancy

366 for species with moderate to high detection probability may not reduce error despite continued

367 effort, while also potentially leading to issues associated with the violation of closure

368 (MacKenzie et al. 2006). Indeed, deriving a biologically meaningful sampling period (e.g.

369 season) during which occupancy status is assumed not to change may vary depending upon the

370 target species and research question, and is therefore a fundamental consideration for survey

371 design (see Gerber, Williams \& Bailey in press). Furthermore, trade-offs in survey approach will

372 be necessary for community-level research, as it is unlikely that a single design will be most

373 efficient for all species. One potential way forward is to initially define the season (timing and

374 scope of inference), and then consider all the species of interest that can be reasonably detected

375 and use the optimum survey effort required to detect all of these taxa (Si et al. 2014). 
377 and occupancy estimates representing a broad spectrum of mammals. The findings can therefore

378 be applied to other taxa and ecosystems where cameras are being used to study small to large

379 terrestrial mammals. To determine an optimal study design, we suggest that researchers first

380 investigate the occupancy (i.e. common, moderately common, rare) and detection (i.e. low,

381 moderate, high) characteristics of their target species. Table 1 and Figure 5 can then be used to

382 guide the required sampling effort (number of sites and survey days) for an acceptable level of

383 error (see also tables provided in MacKenzie \& Royle 2005; Guillera-Arroita, Ridout \& Morgan

384 2010). For many mammals, there may already be published literature in which species occupancy

385 and detection probabilities could be obtained. If no prior information is available, short pilot

386 studies can be very effective in obtaining values for occupancy and detection probability,

387 particularly as these values are often highly site specific, or species experts could be consulted to

388 give a rough estimate regarding occupancy and detection probabilities, depending on body size,

389 behaviour and ranging patterns. Additionally, a recent study has demonstrated a two-stage

390 Bayesian method for incorporating uncertainty in the initial estimates employed in study design

391 (Guillera-Arroita et al. 2014).

392 Finally, as survey effort involves a trade-off between cameras and sampling length, it is

393 important to note that the financial costs associated with these different scenarios may vary

394 considerably. For example, surveying additional sites may require purchasing more cameras,

395 while increasing the survey duration may require personnel to make additional site visits to keep

396 the cameras functioning properly. Under certain scenarios where the number of sites is limiting

397 the accuracy/precision of the estimate and there is sufficient time for two surveys to be conducted

398 within a designated season, cameras can be set for the necessary period and then moved (e.g.,

399 Karanth \& Nichols 2002). Interestingly, this has also proved an efficient method for measuring

400 species richness (Si et al. 2014). It is important to bear in mind that the optimal solution will

401 depend upon the costs associated with camera operation and maintenance versus the costs of

402 procuring cameras (see Table S1\& Spreadsheet S1 for further details on survey design and

403 financial cost).

404 In conclusion, our study investigates the optimal survey effort (sites vs. occasions)

405 required for determining occupancy with a desired level of error across a range of mammalian

406 species using motion-activated cameras. The results of our simulation approach clearly highlight 
407 that simply increasing survey effort is not the most efficient strategy for obtaining a reliable

408 occupancy estimate. The guidelines presented in the paper are based on the analysis of an

409 empirical dataset to provide a real world example that does not solely rely on the simulation of

410 virtual data, while still being directly applicable to research and monitoring programs conducted

411 in other terrestrial ecosystems. We emphasize the use of biological knowledge of the target

412 species coupled with clearly defined a-priori objectives that link monitoring or research effort

413 with defined ecological questions or conservation actions (Martin et al. 2009). Our study also

414 illustrates the value of data simulation approaches for assessing methods and study design before

415 embarking on empirical data collection (Zurell et al. 2010; Ellis, Ivan \& Schwartz 2013).

416 However, it is not always feasible for practitioners to carry out their own simulation exercises.

417 Thus, research such as ours that can provide broad guidelines when a species of interest can be

418 generally classified as rare or common and easily or difficult to detect will be of great utility to

419 designing effective studies.

\section{Acknowledgements}

421 We thank L. Sweanor, B. Dunne, and K. Logan for their invaluable assistance in the field and the

422 landowners who allowed us access to their properties for our research. We are grateful to K.

423 Crooks and L. Bailey for comments on an earlier version of the manuscript and we would like to

424 thank the editor, P. Gandini, and two anonymous reviewers for providing constructive

425 suggestions that greatly improved the manuscript.

\section{References}

427 Ahumada, J.A., Hurtado, J. \& Lizcano, D. (2013) Monitoring the Status and Trends of Tropical

428 Forest Terrestrial Vertebrate Communities from Camera Trap Data: A Tool for Conservation

429 (ed M Somers). PloS one, 8, e73707.

430 Ahumada, J.A., Silva, C.E.F., Gajapersad, K., Hallam, C., Hurtado, J., Martin, E., McWilliam, A., Mugerwa, B., O'Brien, T., Rovero, F., Sheil, D., Spironello, W.R., Winarni, N. \& Andelman, S.J. (2011) Community structure and diversity of tropical forest mammals: data from a global camera trap network. Philosophical Transactions of the Royal Society B: Biological Sciences, 366, 2703-2711.

435 Bailey, L.L., Hines, J.E., Nichols, J.D. \& MacKenzie, D.I. (2007) Sampling design trade-offs in 436 occupancy studies with imperfect detection: examples and software. Ecological Applications, $437 \quad 17,281-290$. 
438

439

440

441

442

443

444

445

446

447

448

449

450

451

452

453

454

455

456

457

458

459

460

461

462

463

464

465

466

467

468

469

470

471

472

473

474 Guillera-Arroita G, Ridout M.S. \& Morgan B.J.T. (2014) Two-stage Bayesian study design for

Betts, M.G., Rodenhouse, N.L., Sillett, S.T., Doran, P.J. \& Holmes, R.T. (2008) Dynamic occupancy models reveal within-breeding season movement up a habitat quality gradient by a migratory songbird. Ecography, 31, 592-600.

Butchart, S.H.M., Walpole, M., Collen, B., van Strien, A., Scharlemann, J.P.W., Almond, R.E.A., Baillie, J.E.M., Bomhard, B., Brown, C., Bruno, J., Carpenter, K.E., Carr, G.M., Chanson, J., Chenery, A.M., Csirke, J., Davidson, N.C., Dentener, F., Foster, M., Galli, A., Galloway, J.N., Genovesi, P., Gregory, R.D., Hockings, M., Kapos, V., Lamarque, J.F., Leverington, F., Loh, J., McGeoch, M.A., McRae, L., Minasyan, A., Morcillo, M.H., Oldfield, T.E.E., Pauly, D., Quader, S., Revenga, C., Sauer, J.R., Skolnik, B., Spear, D., Stanwell-Smith, D., Stuart, S.N., Symes, A., Tierney, M., Tyrrell, T.D., Vie, J.C. \& Watson, R. (2010) Global Biodiversity: Indicators of Recent Declines. Science, 328, 1164-1168.

Di Marco, M., Boitani, L., Mallon, D., Hoffmann, M., Iacucci, A., Meijaard, E., Visconti, P., Schipper, J. \& Rondinini, C. (2014) A Retrospective Evaluation of the Global Decline of Carnivores and Ungulates. Conservation Biology, n/a-n/a.

Ellis, M.M., Ivan, J.S. \& Schwartz, M.K. (2013) Spatially Explicit Power Analyses for Occupancy-Based Monitoring of Wolverine in the U.S. Rocky Mountains. Conservation Biology, 28, 52-62.

Gardner, B., Reppucci, J., Lucherini, M. \& Royle, J.A. (2010) Spatially explicit inference for open populations: estimating demographic parameters from camera-trap studies. Ecology, 91, 3376-3383.

Gerber, B., Karpanty, S.M., Crawford, C., Kotschwar, M. \& Randrianantenaina, J. (2010) An assessment of carnivore relative abundance and density in the eastern rainforests of Madagascar using remotely-triggered camera traps. Oryx, 44, 219.

Gerber, B.D., Ivan, J.S. \& Burnham, K.P. (2014). Estimating the abundance of rare and elusive carnivores from photographic-sampling data when the population size is very small. Population ecology, 56, 463-470.

Gerber, B.D., Williams, P.J. \& Bailey, L.L. (in press) Primates and Cameras. International Journal of Primatology.

Guillera-Arroita, G. \& Lahoz-Monfort, J.J. (2012) Designing studies to detect differences in species occupancy: power analysis under imperfect detection. Methods in Ecology and Evolution, 3, 860-869.

Guillera-Arroita, G., Ridout, M.S. \& Morgan, B.J.T. (2010) Design of occupancy studies with imperfect detection. Methods in Ecology and Evolution, 1, 131-139.

Guillera-Arroita G., Morgan B.J.T., Ridout M.S. \& Linkie, M. (2011) Species occupancy modeling for detection data collected along a transect. Journal of Agricultural, Biological and Environmental Statistics 16: 301-317.

PeerJ reviewing PDF | (v2014:06:2254:1:1:ACCEPTED 31 Jul 2014) 
475

476

477

478

479

480

481

482

483

484

485

486

487

488

489

490

491

492

493

494

495

496

497

498

499

500

501

502

503

504

505

506

507

508

509

species occupancy estimation. Journal of Agricultural, Biological and Environmental Statistics 19: 278-291.

Hamel, S., Killengreen, S.T., Henden, J.-A., Eide, N.E., Roed-Eriksen, L., Ims, R.A. \& Yoccoz, N.G. (2013) Towards good practice guidance in using camera-traps in ecology: influence of sampling design on validity of ecological inferences. Methods in Ecology and Evolution, 4, 105-113.

Jamie, M. (2012) Changing use of camera traps in mammalian field research: habitats, taxa and study types. Mammal Review, 43, 196-206.

Janečka, J.E., Munkhtsog, B., Jackson, R.M., Naranbaatar, G., Mallon, D.P. \& Murphy, W.J. (2011) Comparison of noninvasive genetic and camera-trapping techniques for surveying snow leopards. Journal of Mammalogy, 92, 771-783.

Jones, J.P.G. (2011) Monitoring species abundance and distribution at the landscape scale. Journal of Applied Ecology, 48, 9-13.

Karanth, K.U. \& Nichols, J.D. (2002) Monitoring Tigers and Their Prey: a Manual for Researchers, Managers, and Conservationists in Tropical Asia. Centre for Wildlife Studies.

Kelly, M.J. \& Holub, E.L. (2008) Camera trapping of carnivores: trap success among camera types and across species, and habitat selection by species, on Salt Pond Mountain, Giles County, Virginia. Northeastern Naturalist, 15, 249-262.

Kéry, M. \& Schmid, H. (2004) Monitoring programs need to take into account imperfect species detectability. Basic and Applied Ecology, 5, 65-73.

Larrucea, E.S., Brussard, P.F., Jaeger, M.M. \& Barrett, R.H. (2007) Cameras, Coyotes, and the Assumption of Equal Detectability. Journal of Wildlife Management, 71, 1682-1689.

Long, R.A., Donovan, T.M., MacKay, P., Zielinski, W.J. \& Buzas, J.S. (2010) Predicting carnivore occurrence with noninvasive surveys and occupancy modeling. Landscape Ecology, 26, 327-340.

Mace, G.M. \& Baillie, J.E.M. (2007) The 2010 Biodiversity Indicators: Challenges for Science and Policy. Conservation Biology, 21, 1406-1413.

MacKenzie, D.I., Nichols, J.D., Lachman, G.B., Droege, S., Royle, J.A. \& Langtimm, C.A. (2002) Estimating site occupancy rates when detection probabilities are less than one. Ecology 83, 2248-2255.

MacKenzie, D.I. \& Royle, J.A. (2005) Designing occupancy studies: general advice and allocating survey effort. Journal of Applied Ecology, 42, 1105-1114.

MacKenzie, D.I., Nichols, J.D., Hines, J.E., Knutson, M.G. \& Franklin, A.B. (2003) Estimating site occupancy, colonization, and local extinction when a species is detected imperfectly. Ecology, 84, 2200-2207.

PeerJ reviewing PDF | (v2014:06:2254:1:1:ACCEPTED 31 Jul 2014) 
544 O'Brien, T.G. \& Kinnaird, M.F. (2011) Density estimation of sympatric carnivores using

MacKenzie, D.I., Nichols, J.D., Lachman, G.B., Droege, S., Andrew Royle, J. \& Langtimm, C.A. (2002) Estimating site occupancy rates when detection probabilities are less than one. Ecology, 83, 2248-2255.

MacKenzie, D.I., Nichols, J.D., Royle, J.A., Pollock, K.H., Bailey, L.L. \& Hines, J.E. (2006) Occupancy Estimation and Modeling: Inferring Patterns and Dynamics of Species Occurrence. Elsevier.

MacKenzie, D.I., Seamans, M.E., Gutiérrez, R.J. \& Nichols, J.D. (2010) Investigating the population dynamics of California spotted owls without marked individuals. Journal of Ornithology, 152, 597-604.

Maffei, L., Noss, A.J., Silver, S.C. \& Kelly, M.J. (2011) Abundance/Density Case Study: Jaguars in the Americas. pp. 119-144. Springer Japan, Tokyo.

Magoun, A.J., Long, C.D., Schwartz, M.K., Pilgrim, K.L., Lowell, R.E. \& Valkenburg, P. (2011) Integrating motion-detection cameras and hair snags for wolverine identification. The Journal of wildlife management, 75, 731-739.

Martin, J., Runge, M.C., Nichols, J.D., Lubow, B.C. \& Kendall, W.L. (2009) Structured decision making as a conceptual framework to identify thresholds for conservation and management. Ecological Applications, 19, 1079-1090.

Mattfeldt, S.D., Bailey, L.L. \& Grant, E.H.C. (2009) Monitoring multiple species: Estimating state variables and exploring the efficacy of a monitoring program. Biological Conservation, 142, 720-737.

McDonald-Madden, E., Baxter, P.W., Fuller, R.A., Martin, T.G., Game, E.T., Montambault, J. \& Possingham, H.P. (2010) Monitoring does not always count. Trends in Ecology \& Evolution, 25, 547-550.

Nichols, J.D. \& Williams, B.K. (2006) Monitoring for conservation. Trends in Ecology \& Evolution, 21, 668-673.

Nichols, J.D., Bailey, L.L., O'Connell, A.F., Talancy, N.W., Campbell Grant, E.H., Gilbert, A.T., Annand, E.M., Husband, T.P. \& Hines, J.E. (2008) Multi-scale occupancy estimation and modelling using multiple detection methods. Journal of Applied Ecology, 45, 1321-1329.

Noon, B.R., Bailey, L.L., Sisk, T.D. \& McKelvey, K.S. (2012) Efficient Species-Level Monitoring at the Landscape Scale. Conservation Biology, 26, 432-441.

O'Connell, A.F. \& Bailey, L.L. (2011) Inference for Occupancy and Occupancy Dynamics. pp. 191-204. Springer Japan, Tokyo.

O'Connell, A.F., Nichols, J.D. \& Karanth, K.U. (2011) Camera Traps in Animal Ecology. Springer. 
545

546

547 Ridout, M.S. \& Linkie, M. (2009) Estimating overlap of daily activity patterns from camera trap 548

549 550

551 552

553

554

555

556

557

558

559

560

561

562

563

564

565

566

567

568

569

spatially explicit capture-recapture methods and standard trapping grid. Ecological Applications, 21, 2908-2916. data. Journal of Agricultural, Biological, and Environmental Statistics, 14, 322-337.

Rovero, F. \& Marshall, A.R. (2009) Camera trapping photographic rate as an index of density in forest ungulates. Journal of Applied Ecology, 46, 1011-1017.

Si, X., Kays, R. \& Ding, P. (2014) How long is enough to detect terrestrial animals? Estimating the minimum trapping effort on camera traps. PeerJ, 2, e374.

Thorn, M., Scott, D.M., Green, M., Bateman, P.W. \& Cameron, E.Z. (2009) Estimating Brown Hyaena Occupancy Using Baited Camera Traps. South African Journal of Wildlife Research, 39, 1-10.

Tobler, M.W., Carrillo-Percastegui, S.E., Leite Pitman, R., Mares, R. \& Powell, G. (2008) An evaluation of camera traps for inventorying large- and medium-sized terrestrial rainforest mammals. Animal Conservation, 11, 169-178.

Tyre, A.J., Tenhumberg, B., Field, S.A., Niejalke, D., Parris, K. \& Possingham, H.P. (2003) Improving precision and reducing bias in biological surveys: estimating false-negative error rates. Ecological Applications, 13, 1790-1801.

Webber, A.F., Heath, J.A. \& Fischer, R.A. (2013) Human disturbance and stage-specific habitat requirements influence snowy plover site occupancy during the breeding season. Ecology and Evolution, 3, 853-863.

Yoccoz, N.G., Nichols, J.D. \& Boulinier, T. (2001) Monitoring of biological diversity in space and time. Trends in Ecology \& Evolution, 16, 446-453.

Zurell, D., Berger, U., Cabral, J.S., Jeltsch, F., Meynard, C.N., Münkemüller, T., Nehrbass, N., Pagel, J., Reineking, B., Schröder, B. \& Grimm, V. (2010) The virtual ecologist approach: simulating data and observers. Oikos, 119, 622-635. 


\section{Figure 1}

Location of the study site on the Western Slope, Colorado, USA.

The camera survey was completed in 2009 across 40 grid cells covering $160 \mathrm{~km}^{2}$. 


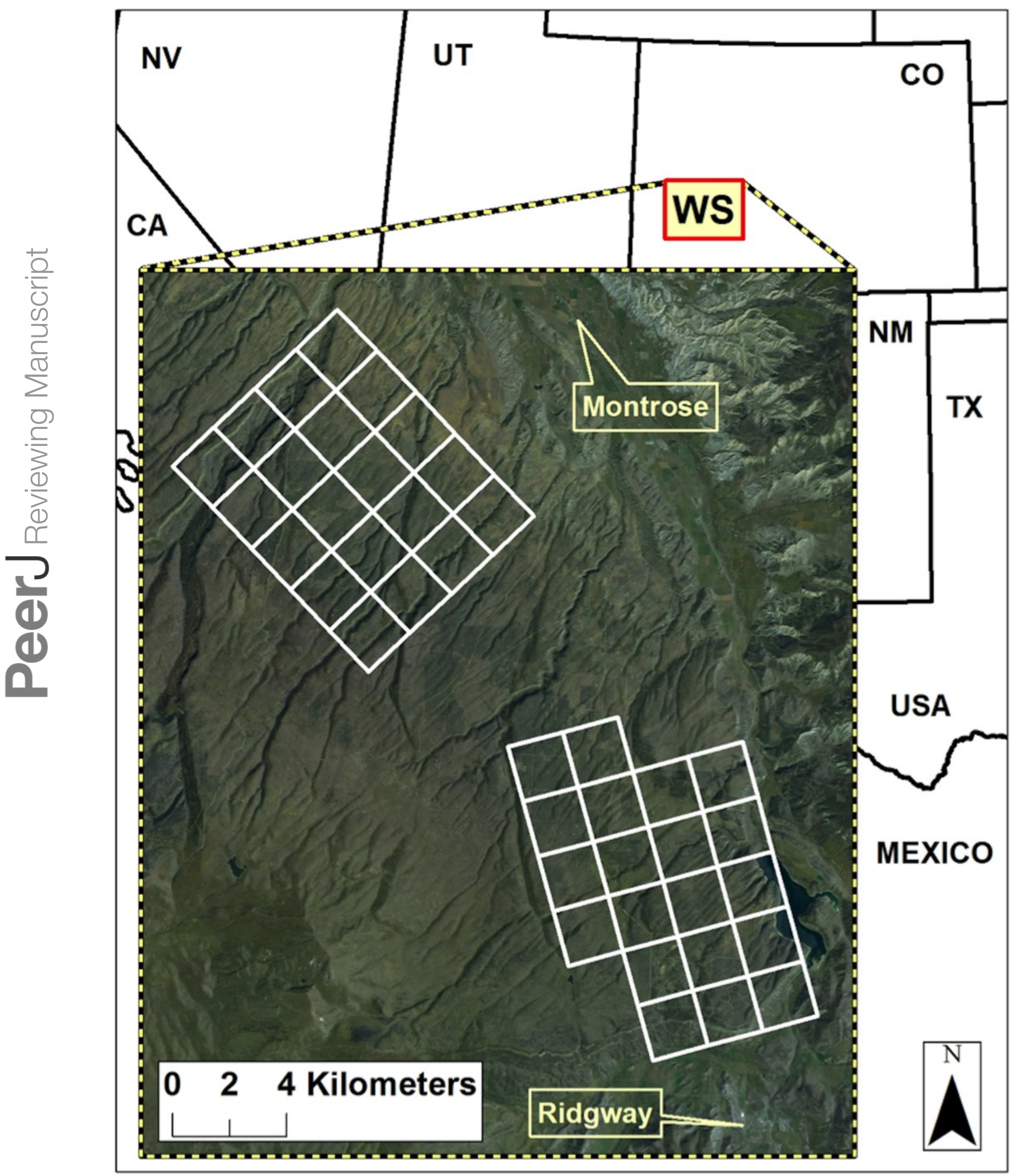




\section{Figure 2}

Motion-activated camera images of mammal species included in the study.

(A) Raccoon, (B) spotted skunk, (C) elk, (D) mountain lion, (E) coyote, (F) bobcat, (G) gray fox, (H) black bear, (I) mule deer and (J) cottontail rabbit (low to high detection probability). 


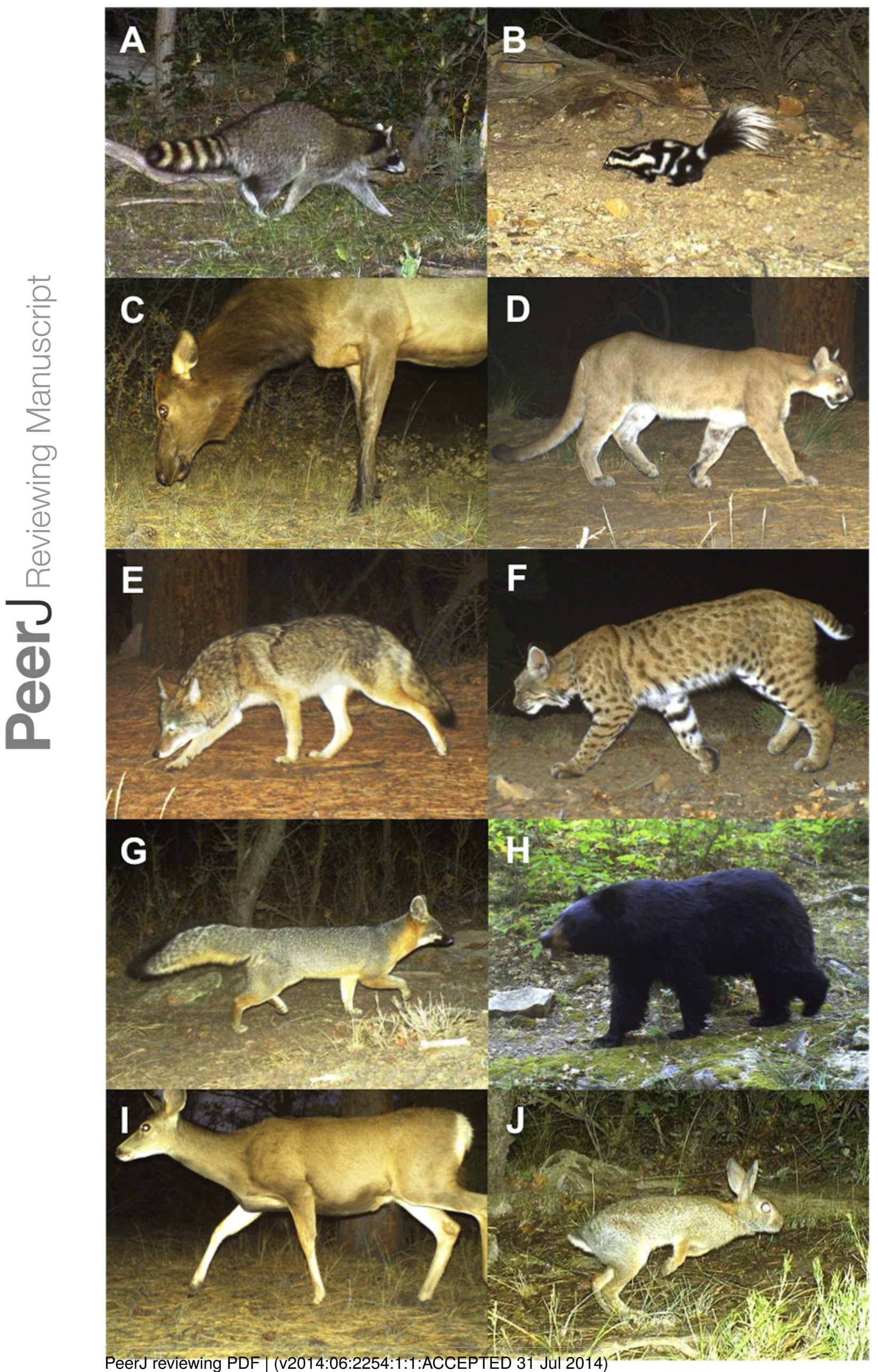




\section{Figure 3}

Occupancy estimates and detection probability for 10-mammals and three virtual species that we used to investigate sampling design trade-offs in a simulation exercise.

The species are grouped according to common characteristics: A) = low occurrence and low detection probability, $\mathrm{B})=$ moderate occurrence and low detection probability, $\mathrm{C}$ ) $=$ high occurrence and low detection probability, D) = moderate occurrence and moderate detection probability, E) = low occurrence and high detection probability, F) $=$ moderate occurrence and high detection probability, G) = high occurrence and high detection probability.

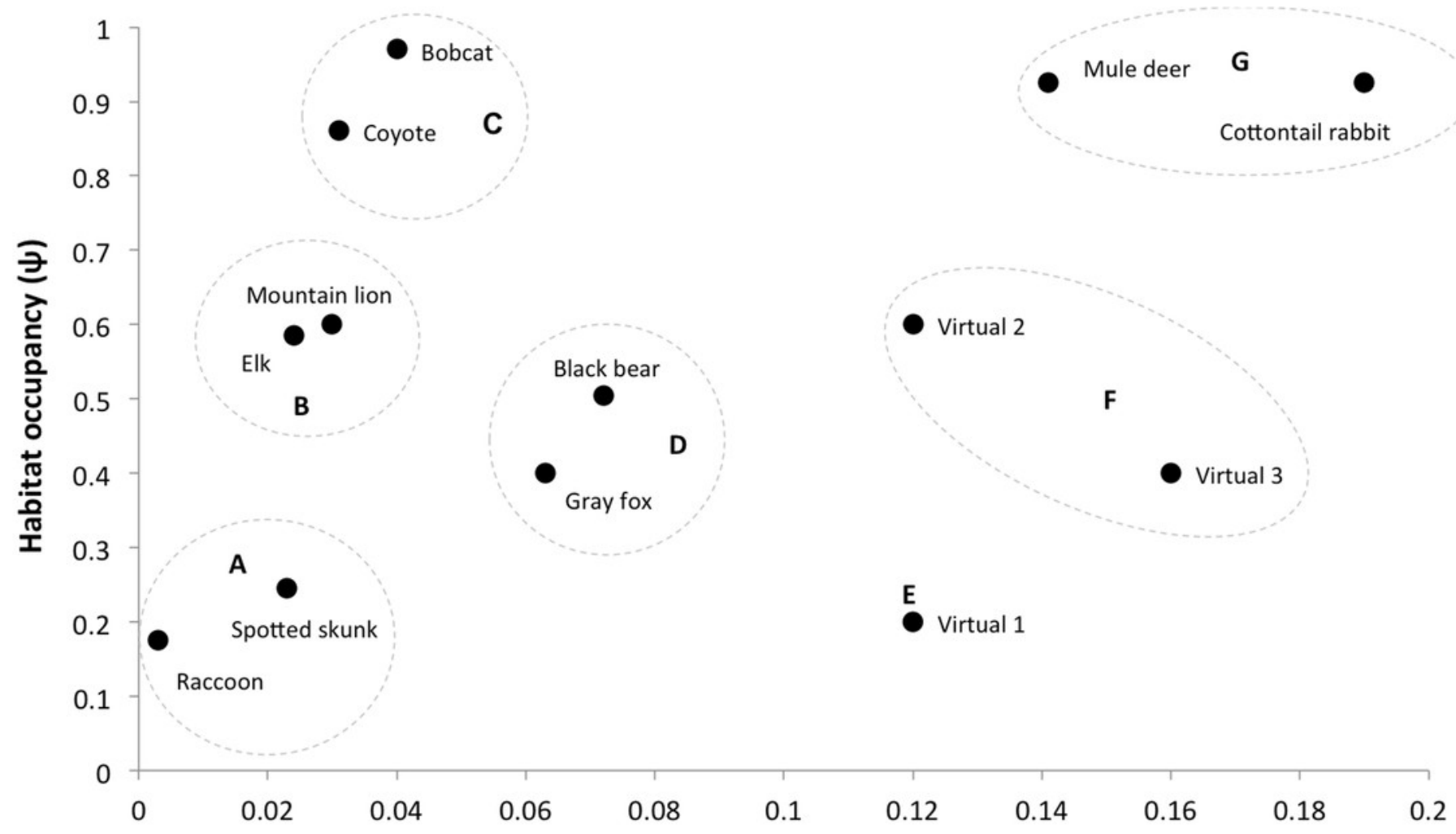

Detection probability (p) 


\section{Figure 4}

Broad recommendations on survey design for studies exploring occupancy using motion-activated cameras.

The symbols indicate high $(+)$, intermediate $(\mathrm{O})$ and low $(-)$ amounts of effort, for the relative number of cameras and survey days to achieve an optimal survey design. From the upperright to the lower-left, an increasing amount of survey effort is required to reliably estimate occupancy.

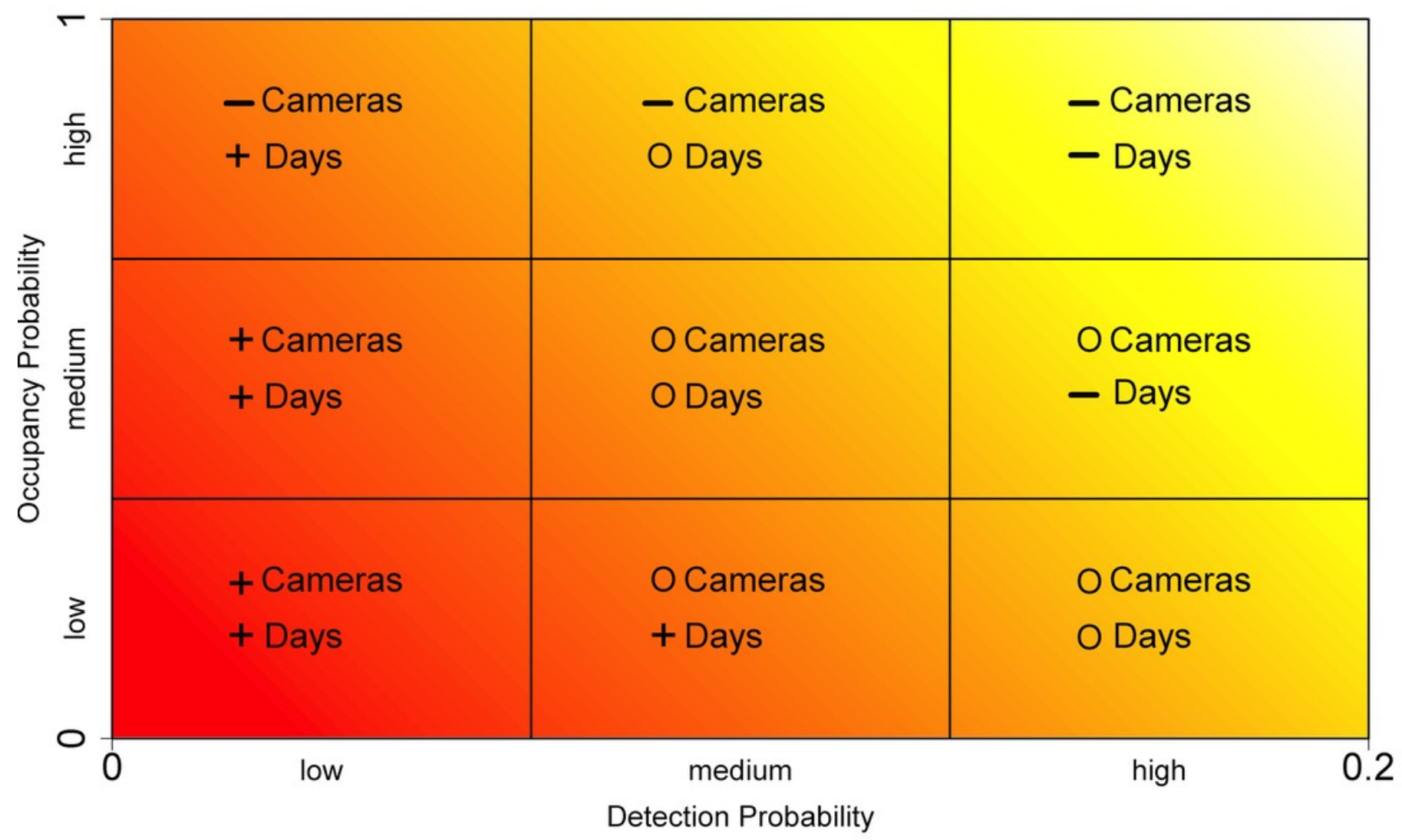




\section{Table $\mathbf{1}_{\text {(on next page) }}$}

Optimal survey design for estimating occupancy with three levels of acceptable error, defined by the root mean squared error (RMSE).

For the purposes of this example we considered the weighting of cameras versus survey days to have the same cost. Raccoons are not included, as a reliable estimate could not be achieved due to the lack of data. 


\begin{tabular}{|c|c|c|c|c|c|c|c|c|}
\hline \multirow{3}{*}{ Species } & \multirow{3}{*}{$\Psi$} & \multirow{3}{*}{$\mathrm{P}$} & \multicolumn{2}{|c|}{ RMSE 0.15} & \multicolumn{2}{|c|}{ RMSE 0.10} & \multicolumn{2}{|c|}{ RMSE 0.075} \\
\hline & & & Sites $^{1} \mathrm{x}$ & Total Survey & Sites $^{1} \mathrm{x}$ & Total Survey & $\operatorname{Sites}^{1} \mathrm{X}$ & Total Survey \\
\hline & & & occasions & effort & occasions & effort & occasions & effort \\
\hline Spotted Skunk & 9.245 & 0.023 & $20 \times 100$ & 2000 & $30 \times 100$ & 3000 & $50 \times 100$ & 5000 \\
\hline Elk & 0.585 & 0.024 & $20 \times 80$ & 1600 & $30 \times 120$ & 3600 & $60 \times 100$ & 6000 \\
\hline Mountain Lion & 0.600 & 0.030 & $20 \times 80$ & 1600 & $30 \times 100$ & 3000 & $60 \times 80$ & 4800 \\
\hline Coyote & 宛 0.861 & 0.031 & $10 \times 60$ & 600 & $20 \times 80$ & 1600 & $30 \times 80$ & 2400 \\
\hline Bobcat & 8.970 & 0.040 & $10 \times 40$ & 400 & $10 \times 60$ & 600 & $10 \times 80$ & 800 \\
\hline Gray Fox & 0.400 & 0.063 & $20 \times 40$ & 800 & $30 \times 40$ & 1200 & $50 \times 40$ & 2000 \\
\hline Black Bear & 0.504 & 0.072 & $20 \times 40$ & 800 & $30 \times 40$ & 1200 & $50 \times 40$ & 2000 \\
\hline Virtual sp. 1 & 0.200 & 0.120 & $20 \times 20$ & 400 & $20 \times 40$ & 800 & $30 \times 60$ & 1800 \\
\hline Virtual sp. 2 & 00.400 & 0.160 & $20 \times 20$ & 400 & $30 \times 20$ & 600 & $50 \times 20$ & 1000 \\
\hline Virtual sp. 3 & 0.600 & 0.120 & $20 \times 20$ & 400 & $30 \times 40$ & 1200 & $50 \times 40$ & 2000 \\
\hline Mule Deer & 0.925 & 0.141 & $10 \times 20$ & 200 & $10 \times 20$ & 200 & $20 \times 20$ & 400 \\
\hline Cottontail Rabbit & 0.925 & 0.190 & $10 \times 20$ & 200 & $10 \times 20$ & 200 & $20 \times 20$ & 400 \\
\hline
\end{tabular}
${ }^{1}$ Sites are the Imber of cameras and occasions are the number of survey days at each site. 


\section{Table 2 (on next page)}

The probability of capturing at least one image of the study species using different survey designs.

The probability $\left(p^{*}\right)$ of detecting a given species at an occupied site at least once over sampling periods of different durations (10-120 occasions). If $\mathrm{p}^{*}$ is close to 1 then detection is effectively perfect for that amount of effort. The shading highlights the number of occasions where $p^{*} \geq 0.9$, and there is only limited improvements in precision to be gained by sampling over longer periods. 


\begin{tabular}{|c|c|c|c|c|c|c|c|}
\hline \multirow[t]{2}{*}{ Species } & \multirow[b]{2}{*}{10} & \multicolumn{6}{|c|}{ Number of sampling occasions } \\
\hline & & 20 & 40 & 60 & 80 & 100 & 120 \\
\hline Raccoon & 0.03 & 0.06 & 0.11 & 0.16 & 0.21 & 0.26 & 0.30 \\
\hline Spotted Skunk & $\stackrel{+}{=} \quad 0.21$ & 0.37 & 0.61 & 0.75 & 0.84 & 0.90 & 0.94 \\
\hline Elk & $\quad 0.22$ & 0.38 & 0.62 & 0.77 & 0.86 & 0.91 & 0.95 \\
\hline Mountain Lion & 0.26 & 0.46 & 0.70 & 0.84 & 0.91 & 0.95 & 0.97 \\
\hline Coyote & 0.27 & 0.47 & 0.72 & 0.85 & 0.92 & 0.96 & 0.98 \\
\hline Bobcat & $\geq \quad 0.34$ & 0.56 & 0.80 & 0.91 & 0.96 & 0.98 & 0.99 \\
\hline Gray Fox & $\underset{\complement}{\subseteq} \quad 0.48$ & 0.73 & 0.93 & 0.98 & 0.99 & 1.00 & 1.00 \\
\hline Black Bear & 0.53 & 0.78 & 0.95 & 0.99 & 1.00 & 1.00 & 1.00 \\
\hline Virtual sp. 1 & 0.72 & 0.92 & 0.99 & 1.00 & 1.00 & 1.00 & 1.00 \\
\hline Virtual sp. 2 & (1) $\quad 0.72$ & 0.92 & 0.99 & 1.00 & 1.00 & 1.00 & 1.00 \\
\hline Virtual sp. 3 & - 0.83 & 0.97 & 1.00 & 1.00 & 1.00 & 1.00 & 1.00 \\
\hline Mule Deer & 0.78 & 0.95 & 1.00 & 1.00 & 1.00 & 1.00 & 1.00 \\
\hline Cottontail Rabbit & 0.88 & 0.99 & 1.00 & 1.00 & 1.00 & 1.00 & 1.00 \\
\hline
\end{tabular}




\section{Figure 5}

Simulation results for each study species.

The influence of survey effort on the error associated with the occupancy estimate (RMSE, root mean squared error), as a function of number of sites (10-120 cameras) occasions (20120 survey days) and species. Species are presented in order of increasing detection probability (from the top left), with the scale of the $y$-axis varying between taxa. Raccoons are absent as a reliable estimate could not be achieved due to the lack of data.

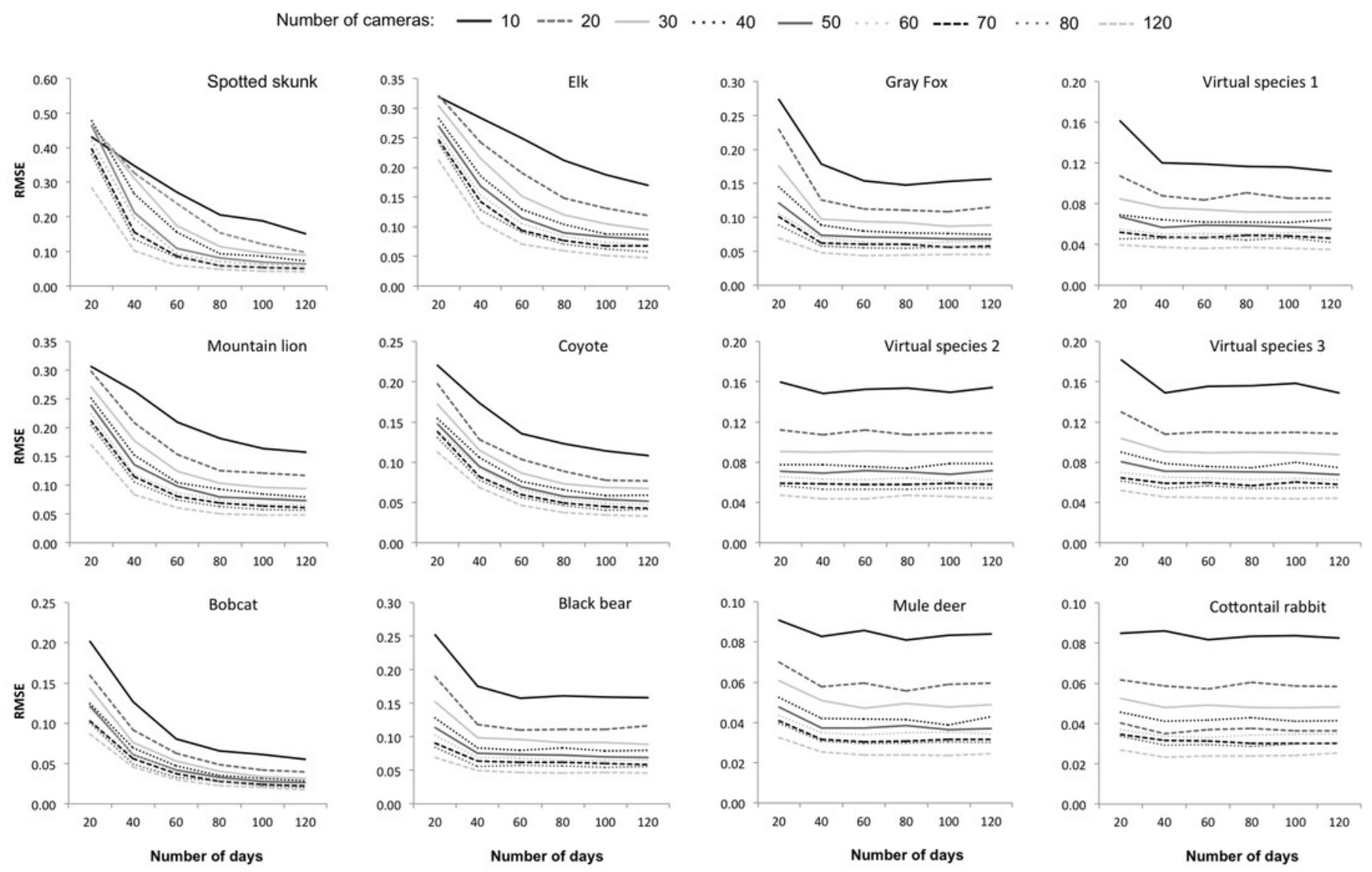

